\title{
Article \\ OFDM PAPR Reduction via Time-Domain Scattered Sampling and Hybrid Batch Training of Synchronous Neural Networks
}

\author{
Ahmad Gendia ${ }^{1,2, * \mathbb{D}}$ and Osamu Muta ${ }^{3}(\mathbb{D}$ \\ 1 Graduate School of Information Science and Electrical Engineering, Kyushu University, \\ Fukuoka 819-0395, Japan \\ 2 Electrical Engineering Department, Faculty of Engineering, Al-Azhar University, Nasr City, \\ Cairo 11751, Egypt \\ 3 Center for Japan-Egypt Cooperation in Science and Technology, Kyushu University, Fukuoka 819-0395, Japan; \\ muta@ait.kyushu-u.ac.jp \\ * Correspondence: ahmad.gendia@mobcom.ait.kyushu-u.ac.jp
}

Citation: Gendia, A.; Muta, O. OFDM PAPR Reduction via Time-Domain Scattered Sampling and Hybrid Batch Training of Synchronous Neural Networks. Electronics 2021, 10, 1708. https:// doi.org/10.3390/electronics10141708

Academic Editor: Alberto Gatto

Received: 3 June 2021

Accepted: 13 July 2021

Published: 16 July 2021

Publisher's Note: MDPI stays neutral with regard to jurisdictional claims in published maps and institutional affiliations.

Copyright: (๑) 2021 by the authors. Licensee MDPI, Basel, Switzerland. This article is an open access article distributed under the terms and conditions of the Creative Commons Attribution (CC BY) license (https:// creativecommons.org/licenses/by/ $4.0 /)$.

\begin{abstract}
Peak-to-average power ratio (PAPR) reduction in multiplexed signals in orthogonal frequency division multiplexing (OFDM) systems has been a long-standing critical issue. Clipping and filtering (CF) techniques offer good performance in terms of PAPR reduction at the expense of a relatively high computational cost that is inherent in the repeated application of fast Fourier transform (FFT) operations. The ever-increasing demand for low-latency operation calls for the development of low-complexity novel solutions to the PAPR problem. To address this issue while providing an enhanced PAPR reduction performance, we propose a synchronous neural network (NN)-based solution to achieve PAPR reduction performance exceeding the limits of conventional CF schemes with lower computational complexity. The proposed scheme trains a neural network module using hybrid collections of samples from multiple OFDM symbols to arrive at a signal mapping with desirable characteristics. The benchmark NN-based approach provides a comparable performance to conventional CF. However, it can underfit or overfit due to its asynchronous nature which leads to increased out-of-band (OoB) radiations, and deteriorating bit error rate (BER) performance for high-order modulations. Simulations' results demonstrate the effectiveness of the proposed scheme in terms of the achieved cubic metric (CM), BER, and OoB emissions.
\end{abstract}

Keywords: clipping and filtering; cubic metric; neural networks; OFDM

\section{Introduction}

The high peak-to-average power ratio (PAPR) is a typical characteristic of orthogonal frequency division multiplexing (OFDM) signals. Prior to transmission through the channel, OFDM signals are amplified, and to achieve high efficiency, the power amplifier is driven near its saturation region. This, however, causes undesired non-linear amplification effects including signal distortion and out-of-band (OoB) radiation, especially if the signal input to the amplifier has high PAPR. Therefore, it is imperative to tackle the high PAPR problem inherent in OFDM signals. Although the PAPR reduction is not the ultimate goal of the communication system, it provides an instrument for maintaining the bit error rate (BER) while keeping the spectral mask at the transmitter output according to standard requirements.

To address the PAPR issue, many classical solutions have been proposed over the years, including partial transmit sequence (PTS) [1], tone reservation (TR) [2], companding [3], selected mapping (SLM) [4], and clipping and filtering (CF) techniques [5-11]. CF is a simple but powerful approach that does not generally require the use of additional resource blocks or the transmission of side information, which make it of particular interest in the literature. In $\mathrm{CF}$, the time-domain OFDM signal amplitude is limited to a predefined threshold level to keep the resulting PAPR within acceptable ranges. However, clipping the 
signal amplitude causes OoB power leakage which may not be tolerable by adjacent channels. The signal is therefore filtered to eliminate the OoB emissions. Nonetheless, filtering is invariably coupled with amplitude regrowth of the time-domain OFDM signal and this in turn increases the PAPR beyond the acceptable threshold. Consequently, iterative-based clipping and filtering (ICF) operation is needed to repeatedly clip the signal amplitude and filter the leaked emissions. However, ICF techniques are computationally expensive in general, especially when the number of sub-carriers is large. Simplified clipping and filtering (SCF) was introduced to achieve similar PAPR reduction performance in a single iteration of modified processing at a relatively cheaper cost. However, the required computational complexity is still high even for SCF.

To solve this problem, the authors in [12] proposed an alternative SCF-based method which uses asynchronous neural network (ANN) modules at the transmitter side to achieve PAPR reduction performance comparable to existing SCF methods while significantly reducing the required complexity. However, the method in [12] is asynchronous in nature due to the separate training of two independent $\mathrm{NN}$ modules using correlated real and imaginary components, respectively. This can potentially cause convergence mismatch between the two NN modules leading, thereby, to one module under or overfitting with respect to the other. This ultimately results in increased OoB radiations and degrading BER performance for higher order modulations. In particular, $\mathrm{OoB}$ radiation is very sensitive to non-linear distortion due to peak limiting. The above inaccurate convergence in conventional ANN may result in unacceptable OoB emissions, which needs a novel solution.

In this paper, we propose a synchronous neural network CF-based (SNN-CF) approach where the two modules of ANN are replaced by a single NN module which is simultaneously fed by both real and imaginary components to take the effect of their interdependence into account at the training stage.

The main contribution of this work can be summarized as follows:

- As an alternative NN-based PAPR reduction approach, we propose a synchronous neural network solution surpassing conventional CF-based approaches. The proposed method accurately generates CF-like output signals by synchronously learning both real and imaginary parts of raw OFDM input signals and mapping them to the desired target output. Hence, higher PAPR reduction performance and lower OoB power leakage can be achieved compared to conventional ANN solutions.

- To find a neural network with desirable characteristics, we propose a networkselection algorithm to explore a wide variety of candidate solutions during the training stage. The algorithm scans the neighborhood of conventional SCF or ICF searching for the mapping network with the highest PAPR reduction for a maximum allowable BER threshold.

- To prevent overfitting traps and arrive at a network model that generalizes well to new data during deployment, we propose an algorithm for the compilation of a 3-D training dataset that randomly scatters the training samples of the same OFDM symbol across multiple different 2-D slices within the dataset. Thus, hybrid OFDM symbols are constructed from the time-domain samples of multiple different raw OFDM symbols during the training stage.

- Through the numerical evaluation of the system performance, we show that the proposed SNN-CF scheme achieves superior performance in terms of PAPR reduction capability, while exhibiting less OoB radiation for both QPSK and 16-QAM compared with ANN. Moreover, the proposed scheme maintains adequate BER levels for higher order modulations such as 16-QAM, where the asynchronous approach shows rapid BER degradation.

Notation: $|$.$| and [.]^{T}$ denote the absolute and transpose operators, respectively. $\mathcal{R}\{\},. \mathcal{I}\{$.$\} , and \angle($.$) denotes the real part, imaginary part, and radian angle information$ of their arguments, respectively. $\overline{(.)}$ denotes the expectation operation. $0_{a, b, c}$ denotes an $a$-by-b-by-c matrix of all zeros. 


\section{Related Works}

The authors in [13] investigated the modeling of the PAPR reduction problem as a combinatorial search problem and proposed to use a Hopfield neural network (HNN) optimizer [14] to solve it. Although the technique showed promising results, it would require the transmission of a large amount of side information and suffer high sensitivity to phase noise. To achieve PAPR reduction performance close to that provided by HNN while avoiding the transmission of side information, an SLM-based radial basis function network (RBFN) solution was introduced in [15]. RBFN is essentially a multivariate interpolator that generates a phase rotation vector which is fed to an SLM encoder for phase selection and OFDM signal generation. Although no explicit side information is needed, the technique requires the adaptive updating of the weights of the network's output layer as well as the activation functions of the network's hidden layers.

The authors in [16] proposed an active constellation extension-based (ACE) method that deploys a time-frequency neural network (TFNN) to accomplish PAPR reduction performance close to that obtainable using the original ACE technique with less complexity. However, TFNN modules operate in complex time-domain and frequency-domain modes and exhibit degraded BER performance in fading channels for high-order modulations. To overcome this problem, Ref. [17] proposed an ACE-based NN variation to achieve much better BER performance for high-order modulation transmissions over fading channels while maintaining almost the same PAPR reduction performance of the TFNN method. Although the technique requires fewer in-use computations than the TFNN approach, it would rely on the continuous training and updating of adaptive NN modules at the receiver side, based on the average received signal-to-noise (SNR) ratio, through the transmission of predefined training signals over dedicated spectral resources.

Recently, the authors in [18] proposed a deep neural network-based (DNN) encoders and decoders deploying multi-layer DNN modules for constellation mapping and demapping at the transmitter and receiver sides, respectively. The DNN-based approach can simultaneously improve the performance in terms of both PAPR reduction and BER. This comes, however, at the expense of highly expensive computational cost due to the deployment of complex multi-layer DNNs, rendering their usage unfeasible for low-latency demanding scenarios that require strict constraints on complexity.

Unlike the above works, the proposed SNN-CF is a low-complexity PAPR reduction approach which requires neither the active adaptation of the learned network nor the transmission of side information or the dedication of additional resource blocks. In addition, the actual merit of the proposed scheme is expected to be observed for higher-order modulations where conventional ANN schemes start to exhibit severe BER performance deterioration.

\section{OFDM Model}

\subsection{Signal Model}

Consider an OFDM system utilizing $N$ sub-carriers. In order to closely follow the continuous amplitude envelope of the time-domain OFDM signal, an oversampling factor $\eta \geq 4$ is customarily assumed. The time-domain OFDM signal can be written as

$$
x[n]=\frac{1}{\sqrt{\eta N}} \sum_{k=1}^{\eta N} X[k] e^{\frac{\omega_{k} n}{\eta N}} \forall n=1,2, \ldots, \eta N,
$$

where $\omega_{k}=2 \pi k$ is the $k$-th sub-carrier carrying the data-bearing modulated symbol $X[k]$. Quadrature phase shift keying (QPSK) modulation and quadrature amplitude modulation (16-QAM) are both considered subsequently.

\subsection{Performance Metric}

As mentioned earlier, high-power amplifiers (HPAs) have non-linear responses causing undesired distortion effects which are poorly captured by the classical peak-to-average power ratio metric. The cubic metric (CM) [19] uses higher-order statistical information 
gleaned from the OFDM signal to appropriately account for the signal's envelope fluctuations. The third-generation partnership project (3GPP) defines the $\mathrm{CM}, \Omega$, as

$$
\Omega=\frac{\Gamma^{[d B]}-\Gamma_{r e f}^{[d B]}}{\kappa} d B
$$

where $\Gamma^{[d B]}$ is the raw cubic metric (RCM) of the time-domain OFDM signal in (1) and is defined as

$$
\Gamma^{[d B]}=20 \log _{10}\left\{\sqrt{\left(\left[\frac{|x[n]|}{\sqrt{\overline{x[n]}}]^{3}}\right)\right.}\right\} d B,
$$

where the factor $\kappa$ is empirically determined and $\Gamma_{r e f}^{[d B]}$ is a fixed RCM reference level. Typical values for these two parameters are used later in the numerical evaluation of the system performance.

\section{Benchmark Techniques}

\subsection{Selected Mapping}

Undistorting PAPR reduction schemes, such as SLM, sacrifice complexity or the utilization of additional resources to help maintain the same BER performance of the original OFDM. In SLM [4], a large block of $U$ different OFDM sequences, $x_{u}[n] \forall u=1,2, \ldots, U$, $n=1,2, \ldots, \eta N$, representing alternative candidates to map the same information-bearing symbol stream, is generated. The candidate sequence, $x^{*}[n]$, with the lowest CM metric, is selected for transmission. Thus, for SLM:

$$
x^{*}[n]=\underset{\substack{x_{u}[n] \\ u \in \mathcal{S}}}{\arg \min } \frac{20 \log _{10}\left\{\sqrt{\left(\left[\frac{\left|x_{\mathcal{u}}[n]\right|}{\overline{\overline{\bar{x}_{u}[n]}}}\right]^{3}\right)}\right\}-\Gamma_{r e f}^{[d B]}}{\kappa},
$$

where $\mathcal{S} \triangleq\{1, \ldots, U\}$ is the set of indices of all candidate sequences within the generated block.

\subsection{Clipping-Based Techniques}

\subsubsection{Iterative Clipping and Filtering}

The ICF algorithm is outlined in Figure 1. The signal amplitude is kept below a preconfigured limiting threshold by repeatedly clipping amplitudes exceeding the threshold and filtering the resulting OoB radiations. The process therefore zigzags back and forth between time and frequency domain representations through the iterative computation of IFFT and FFT operations for a preset number of times, $L$. Fortunately, amplitude regrowth decays with the increasing number of iterations, thereby enabling the technique to effectively reduce the PAPR level as more iterations are applied.

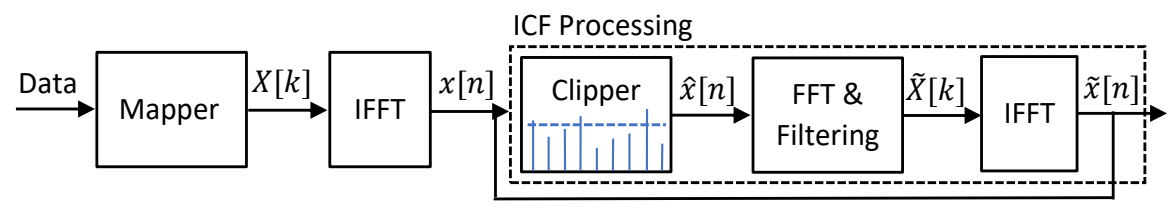

Figure 1. Top-level view of ICF.

\subsubsection{Simplified Clipping and Filtering}

SCF mimics the powerful PAPR reduction capability of the ICF-based mechanism while dispensing the need for iterative operation. As in ICF, SCF algorithm [8] starts by applying IFFT operation to generate the time-domain OFDM signal, $x[n]$, from the modulated symbol stream sequence, $X[k]$, and then proceeds to compute a clipped version, 
$\hat{x}[n]$, with maximum amplitude $R$, according to the same criterion. However, the operation is then modified to achieve almost the same performance in a single iteration of signal processing. The clipped signal, $\hat{x}[n]$, is then subtracted from the unclipped OFDM signal, $x[n]$, to produce a clipping noise signal, $d[n]$, which is then converted to frequency-domain representation, $D[k]$, by applying FFT operation as shown in Figure 2. To suppress OoB emissions, $D[k]$ is then filtered to yield $\hat{D}[k]$ with nulled $(\eta-1) N$ trailing components. A crucial step giving SCF its ability to come very close to ICF performance without the need for multiple iterations was then performed. The frequency-domain clipping of the original modulated sequence, $X[k]$, is accomplished by scaling the filtered noise signal, $\hat{D}[k]$, and subtracting it from $X[k]$, to obtain $\tilde{X}[k]$ which is finally converted to the time domain sequence $\tilde{x}[n]$ by applying IFFT operation. The clipped signal, $\tilde{X}[k]$, was computed as

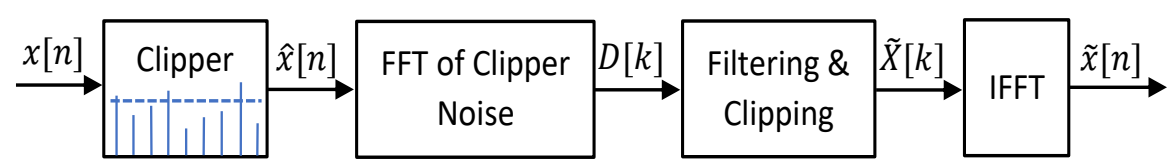

Figure 2. Top-level view of SCF.

$$
\tilde{X}[k]=X[k]-\rho \hat{D}[k],
$$

where the scaling coefficient, $\rho$, is calculated as

$$
\rho=\frac{1-(1-\mu)^{1.5 \lambda}}{1-(1-\mu)^{1.5}},
$$

where the factor $\lambda$ was used to establish an equivalency for the iterative nature of ICF and takes the same value as the number of iterations used by ICF. The parameter, $\mu$, is a function of the maximum allowable amplitude, $R$, and the standard deviation, $\sigma$, of the unclipped time-domain OFDM signal, $x[n]$, and is computed as

$$
\mu=\sqrt{\frac{8}{3 \pi}}\left(\frac{\sigma}{R}\right) .
$$

\section{Proposed Neural Network Solution}

\subsection{Network Architecture}

The objective is to train a neural network to extract the identifying characteristics inherent in time-domain OFDM signals processed by conventional CF-based solutions. A feed-forward multi-layer perceptron (FFMLP) neural network is pretrained offline before it is deployed for the real-time processing of original OFDM signals prior to transmission. As shown in Figure 3a, the general structure of FFMLP consists of three main sections: an input layer defining the input format (type, dimensionality, etc.,) followed by a set of hidden layers, each including a configurable number of neurons with a unified activation function for all neurons of each layer. The last layer is the output layer, which is a convergence point collecting the data traversing the network from the input layer through the hidden layers. The data are weighted and summed by the output layer's neurons and passed through an activation function to produce the final output. The input to the FFMLP is $M$-dimensional. There is a total of $V$ hidden layers. The $i$-th hidden layer contains $h_{i}$ processing neurons. The final output of the FFMLP network module is $P$-dimensional. The response of the $j$-th neuron in the $i$-th hidden layer is:

$$
s_{j}^{(i)}=f_{i}\left(\sum_{k=1}^{h_{i-1}} w_{j, k}^{(i)} s_{k}^{(i-1)}+b_{j}^{(i)}\right),
$$


where $f_{i}($.$) is the activation function of the i$-th hidden layer and $s_{k}^{(0)} \triangleq I_{k}$ is the $k$-th entry in the input layer. $w_{j, k}^{(i)}$ is the learnable weight connecting the $k$-th neuron in the $(i-1)$-th layer to the $j$-th neuron in the $i$-th layer. $b_{j}^{(i)}$ is the learnable bias of the $j$-th neuron in the $i$-th layer. Similarly, the response of the $j$-th neuron in the output layer is:

$$
s_{j}^{(o)}=f_{o}\left(\sum_{k=1}^{h_{V}} w_{j, k}^{(o)} s_{k}^{(V)}+b_{j}^{(o)}\right) .
$$

Figure $3 \mathrm{~b}$ illustrates the application of an FFMLP module to process raw OFDM input signals. The input layer extracts the in-phase and quadrature components of the input OFDM signal. The two parts are then simultaneously processed by the subsequent core of the pretrained neural network module to generate the corresponding in-phase and quadrature components of a CF-like output signal with the desired characteristics.

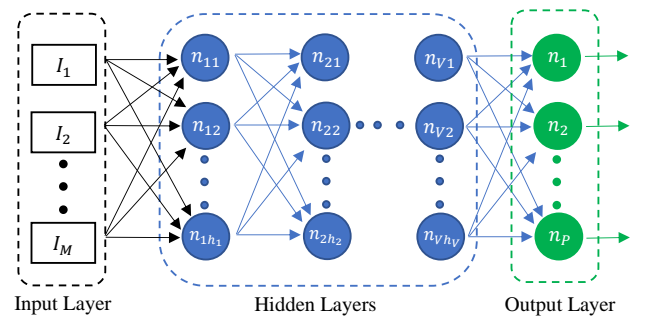

(a)

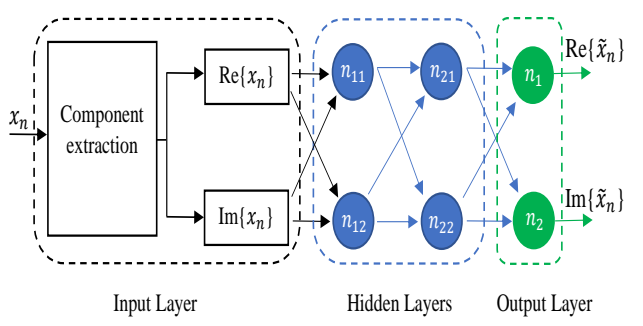

(b)

Figure 3. FFMLP neural networks: (a) general structure; and (b) OFDM signal processing.

\subsection{Proposed Dataset Compilation and Network Selection}

A 3-D training dataset, $\mathcal{D}$, is formed by running Algorithm 1. Initially, $\mathcal{D}$ is propagated by zeros. The dataset, $\mathcal{D}$, is comprised of multiple 2-D slices as shown in Figure 4 . The $i$ th 2-D slice, $\mathcal{D}(i, .,.) \forall i=1,2, \ldots, S$, is filled with a random selection of a group of $\eta N$ time-domain samples. The samples are obtained by generating a vector of samples of an original OFDM symbol, $x_{i}[n] \forall n=1,2, \ldots, \eta N$, which is then fed to an ICF-based clipping algorithm to generate the corresponding output training vector, $x_{o}[n] \forall n=1,2, \ldots, \eta N$. SCF-based training can be applied alternatively. The samples of the two generated vectors are jointly scattered across the slices in a random fashion as shown in Figure 4 . The process is repeated until all the slices within $\mathcal{D}$ are filled with the training data.

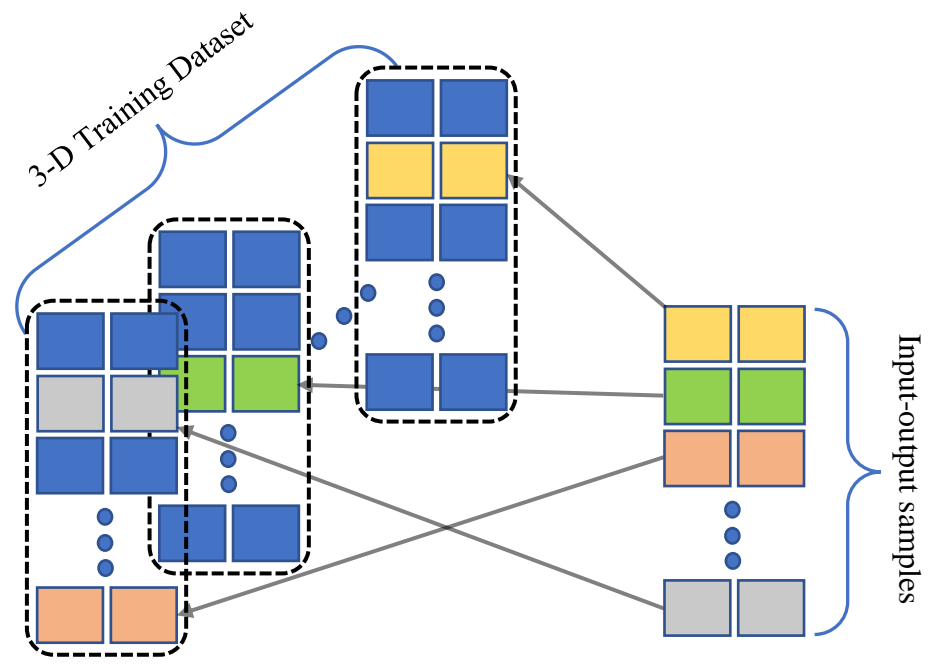

Figure 4. Proposed dataset compilation mechanism. 
Figure 5 illustrates the concept of the proposed SNN-CF scheme. Unlike conventional NN solutions, we propose using a synchronous FFMLP-based network structure to achieve a more accurate performance with a slight increase in computational cost. In this scheme, instead of processing the correlated (I) and (Q) parts independently, only one FFMLP module is employed to process both parts simultaneously, using 2-dimensional (2-D) input and output sequences $(M=2$ and $P=2$ in Figure 3 a). This synchronous approach can emulate SCF with higher flexibility and handle underfitting and overfitting problems more gracefully than ANN where two modules are trained separately. Therefore, SNN-CF essentially models $\mathcal{R}\{\tilde{x}[n]\}$ as a function of both $\mathcal{R}\{x[n]\}$ and $\mathcal{I}\{x[n]\}$. Similarly, $\mathcal{I}\{\tilde{x}[n]\}$ is modeled as a function of both $\mathcal{R}\{x[n]\}$ and $\mathcal{I}\{x[n]\}$. Thus, SNN-CF has potentially higher chances of capturing the essence of the SCF peak-limiting mapping algorithm.

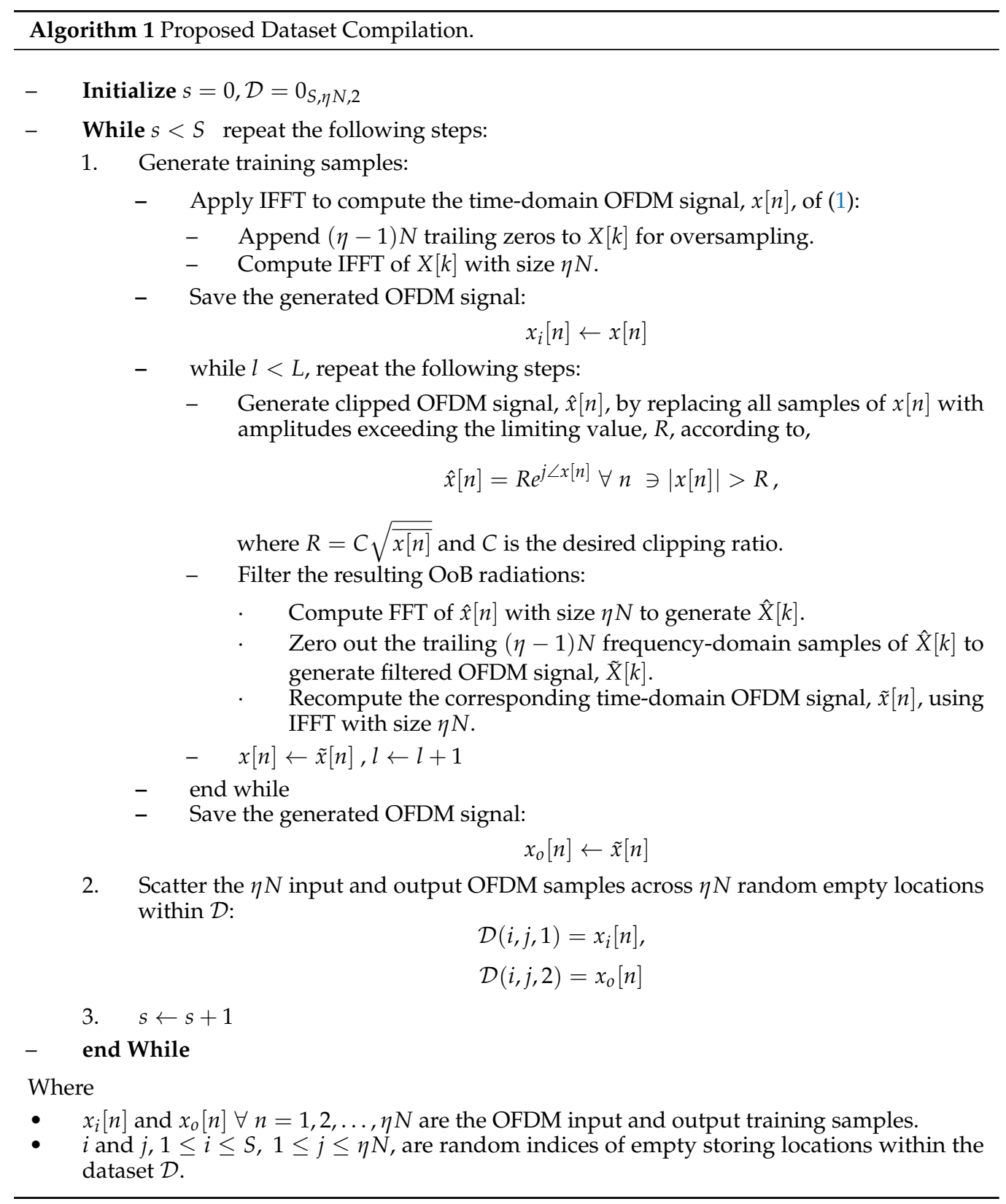




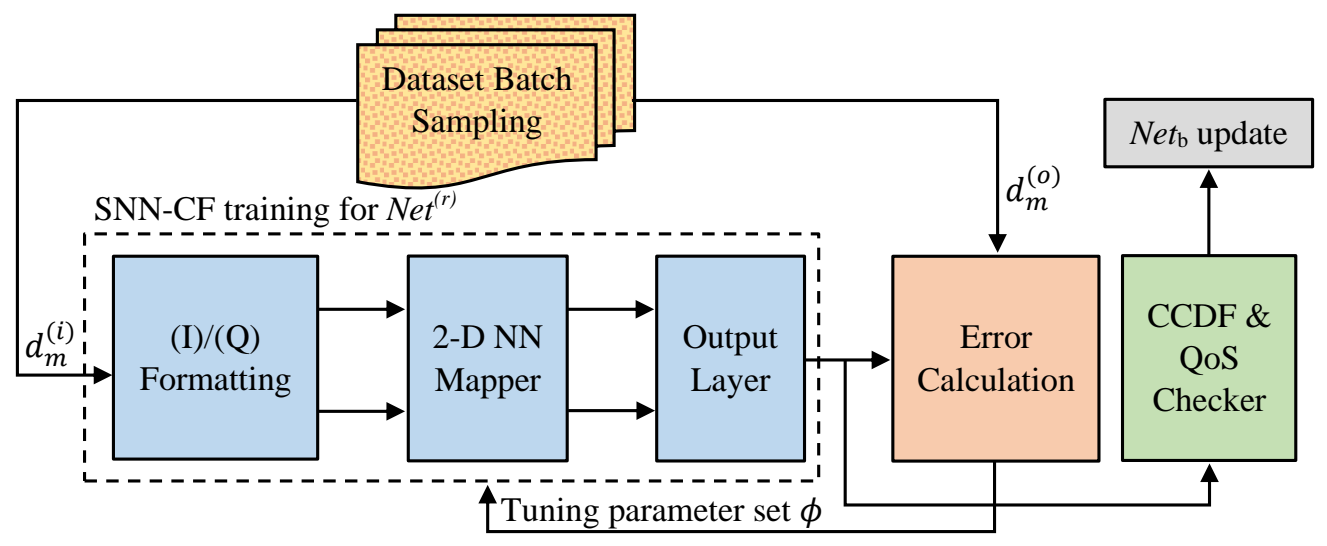

(a)

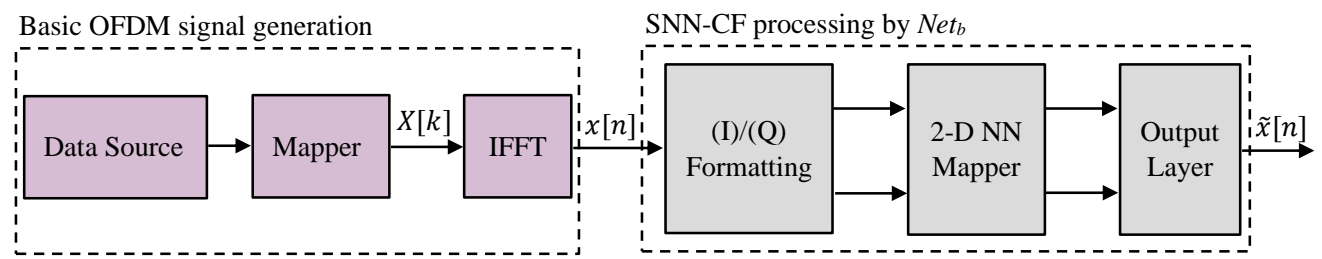

(b)

Figure 5. The proposed scheme: (a) training; and (b) deployment.

During training the network model in Figure 5, each training data point is formed from $\mathcal{D}$ by constructing an input training vector, $[\mathcal{R}\{\mathcal{D}(i, j, 1)\}, \mathcal{I}\{\mathcal{D}(i, j, 1)\}]^{T}$, and a corresponding target vector, $[\mathcal{R}\{\mathcal{D}(i, j, 2)\}, \mathcal{I}\{\mathcal{D}(i, j, 2)\}]^{T}$. The training process goes on until the mean square error (MSE) between the actual output of the NN module and the target output is minimized. An SNN-CF network is found once the training process halts. The network is then ready and can be deployed for the processing of raw OFDM signals that it has not processed before while learning SCF patterns. Compared to ANN, the proposed SNN-CF scheme is able to converge within fewer iterations during the training stage.

When deployed for operation, SNN-CF starts by reformatting the original OFDM input $x[n]$ to produce $[\mathcal{R}\{x[n]\}, \mathcal{I}\{x[n]\}]^{T}$, a 2-D vector representation of the in-phase and quadrature components of $x[n]$. The input vector is then applied to the network's Hidden Layers section which is preconfigured during the training stage to implement a map $f: \mathbb{R}^{2} \rightarrow \mathbb{R}^{2}$ in the neighborhood of conventional ICF or SCF. The output layer processes the 2-D signal vector produced by the vector mapper $f$ to generate a close estimate to $\tilde{x}[n]$, which would be the output of conventional ICF or SCF for the same input $x[n]$.

Attaining a higher performance than conventional CF-based schemes is accomplished by exploring the space of different maps in the neighborhood of ICF or SCF. This is performed by repeatedly training different SNN-CF modules until a network providing the highest positive performance gap is found under certain quality of service (QoS) requirements. Thus, to ensure the fidelity of the solution, we impose a threshold BER, $\mathcal{E}_{t}$, for the trained SNN-CF network at an arbitrary $E_{b} /\left.N_{0}\right|_{t}$. Therefore, in order to find a mapper $f$ with desirable characteristics, we explore the neighborhood of a conventional CF map during the offline training stage. The outline of the proposed network-selection algorithm is given in Algorithm 2. The basic concept of the algorithm is to optimize the network structure to achieve the lowest possible CM value while maintaining a given QoS requirement. Although a maximum allowable BER level is used as a metric to enforce the required QoS, other metrics can be applied, such as the sum-rate and error vector magnitude (EVM). In fact, EVM is used as a metric to achieve the target BER in [20]. The algorithm starts by initializing an SNN-CF network, Net ${ }^{(0)}$, with a random parameter set $\phi$ and updates it using a random batch of training data points from the dataset $\mathcal{D}$ in order to find a mapping solution that minimizes the MSE mentioned previously. For a 
preset number of iterations, different SNN-CF networks are generated. In each iteration, $r$, a different non-linear map in the neighborhood of ICF or SCF is learned by training a new candidate SNN-CF network, Net ${ }^{(r)}$. Upon training, the performance of Net $t^{(r)}$ is measured by evaluating $\mathcal{F}^{(r)} \triangleq \operatorname{Pr}\left\{\Omega>\Omega_{0 d}\right\}$, the complementary cumulative distribution function (CCDF) of the OFDM signal generated by $\mathrm{Net}^{(r)}$. As the iterations advance, the algorithm keeps track of Net ${ }_{b}$, the SNN-CF network providing the best CCDF value, $\mathcal{F}_{b}$. The best-performing network is thus updated and saved as the algorithm progresses. Finally, the algorithm outputs the best-performing SNN-CF network found when it stops after the last iteration.

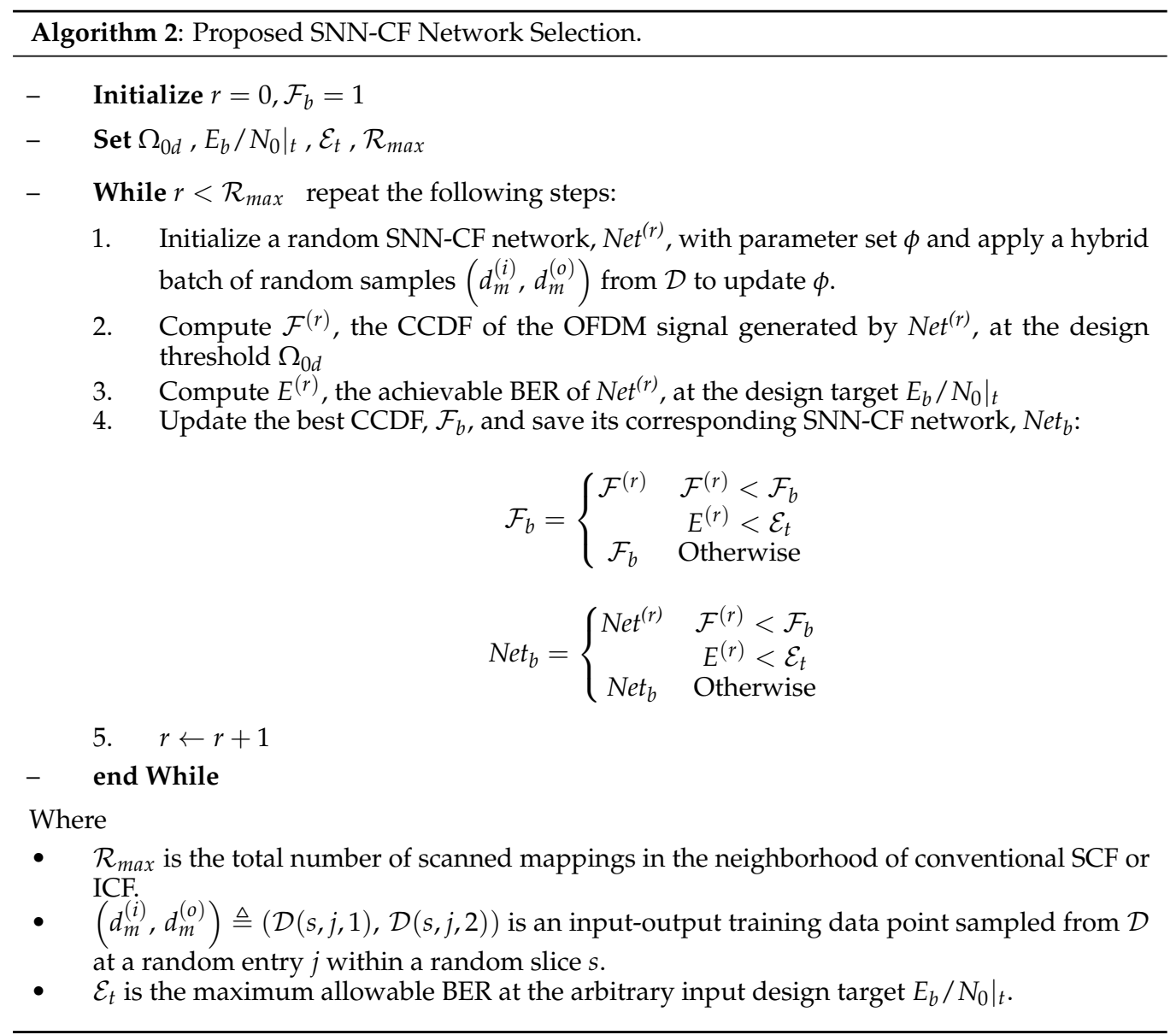

\section{Complexity Analysis}

In this section, we discuss the online computational requirements of the proposed scheme compared to the conventional solutions as well as ANN approach. We focus on the on-line computational demand since the training algorithms are offline-based, rendering the deployment phase as the critical stage for computation. SLM performs the highest amount of computations and generally requires $U$ IFFT operations, $U C M$ computations and $U$ check operations for the selection of the sequence having the lowest $\mathrm{CM}$ value. For all other schemes, the time-domain OFDM signal is first generated through the application of an IFFT operation to the modulated data sequence, $X[k] \forall k=1,2, \ldots, \eta N$, which typically requires $(N / 2) \log _{2}(N)$ complex multiplications and $N \log _{2}(N)$ complex additions.

Each iteration of the ICF algorithm requires one clipping operation followed by an FFT operation to zero out the frequency-domain OoB radiations and finally an IFFT operation to convert back to the time domain. ICF thus requires $L$ clipping operations as well as a total of $2 \mathrm{LNlog}_{2}(N)$ and $\mathrm{LNlog}_{2}(N)$ complex addition and multiplication operations, respectively. SCF processes the signal in a single iteration and requires one 
clipping operation and a total of $N\left(2 \log _{2}(N)+2\right)$ and $N\left(\log _{2}(N)+1\right)$ complex addition and multiplication operations, respectively.

Each module of ANN performs $2 \mathrm{~N}$ real multiplications and $2 \mathrm{~N}$ real additions in each hidden layer as well as $N$ real multiplications and $N$ real additions in the output layer. Thus, ANN requires a total of $10 \mathrm{~N}$ real multiplications and $10 \mathrm{~N}$ real additions. The output layer as well as each hidden layer of the proposed SNN-CF scheme performs $4 \mathrm{~N}$ real multiplications and $4 \mathrm{~N}$ real additions, resulting in a total of $12 \mathrm{~N}$ real multiplications and $12 \mathrm{~N}$ real additions. Finally, unlike conventional approaches, neither ANN nor SNN-CF requires additional checking or clipping operations. Therefore, neural network-based approaches provide significantly less computation requirements in general, rendering the real-time operation of such schemes much more favorable.

The computational cost of the investigated schemes are summarized in Table 1. The complexity of both ANN and the proposed SNN-CF schemes are based on computations in $\mathbb{R}$, the set of real numbers. However, the remaining schemes operate in the complex domain. Since one complex addition is equivalent to two real additions and one complex multiplication is equivalent to four real multiplications and two real additions, the equivalent complexities of ANN and SNN-CF are $\mathcal{O}(6.67 N)$ and $\mathcal{O}(8 N)$, respectively. Although compared to ANN the proposed SNN-CF scheme requires a slight increase in computational cost within the same asymptotic bound on complexity, it can potentially boost the system performance in terms of PAPR reduction and better controlled OoB emissions while simultaneously providing a lower BER for high-order modulations.

Table 1. Complexity requirements of the investigated schemes.

\begin{tabular}{cc}
\hline Scheme & Complexity \\
\hline SLM [4] & $\mathcal{O}\left(U\left(1.5 N \log _{2} N+N+1\right)\right)$ \\
\hline ICF [5] & $\mathcal{O}\left(L N\left(3 \log _{2} N+1\right)\right)$ \\
\hline SCF [8] & $\mathcal{O}\left(N\left(3 \log _{2} N+4\right)\right)$ \\
\hline ANN [12] & $\mathcal{O}(20 N)$ \\
\hline Proposed SNN-CF & $\mathcal{O}(24 N)$ \\
\hline
\end{tabular}

\section{Numerical Results and Discussion}

\subsection{Simulation Setup}

The parameters of the simulation environment are configured as in Table 2. We assume an OFDM system utilizing $N=256$ sub-carriers loaded with QPSK or 16-QAM data. An oversampling factor $\eta=4$ was chosen for the accurate tracking of the timedomain signal envelope. The RCM of the reference signal is simulated at $1.52 \mathrm{~dB}$ and the empirical factor $\kappa$ is fixed at the value of 1.56 for all simulated schemes. The clipping ratio defining the maximum allowable amplitude of the time-domain OFDM signal processed by ICF and SCF schemes is set to $6 \mathrm{~dB}$. Three ICF iterations are used and the equivalency factor used in SCF is set to the same value. The signal's standard deviation parameter $\sigma$ is set to $1 / 2$ for the proper estimation of the frequency-domain scaling coefficient $\rho$. The block size representing the total number of alternative sequences in SLM is set to 16.

The feed-forward multi-layer perceptron NN architecture model is adopted in both ANN and the proposed SNN-CF scheme due to its simplicity and robustness in accurately implementing various mappings in the processing of wireless communication signals. ANN uses two NN modules with two hidden layers in each module. The first layer has two neurons and the second layer has one neuron. The activation function used in both hidden layers of ANN is the triangular function defined as $f(x) \triangleq 1-|x|,|x| \leqslant 1$, and $f(x) \triangleq 0$ otherwise.

A design target $E_{b} /\left.N_{0}\right|_{t}$ is defined at the input of the PAPR reduction where the achievable BER is confined to a limiting threshold $\mathcal{E}_{t}$. The proposed SNN-CF scheme deploys a single NN module which has two hidden layers. Both layers have two neurons 
each. The activation function used to transfer the weighted input plus bias from the input terminal of a neuron to its output terminal is the hyperbolic tan-sigmoid transfer function defined as $g(x) \triangleq 2 /\left(1+e^{-2 x}\right)-1 \forall x \in \mathbb{R}$. Both ANN and the proposed SNN-CF scheme use the purelin activation function, $h(x) \triangleq x \forall x \in \mathbb{R}$, for the output layer's neurons. The design threshold $\Omega_{0 d}$ used in Algorithm 2 for SNN-CF network selection is arbitrary and is set to $3 \mathrm{~dB}$ for both QPSK and 16 QAM cases. In addition, the maximum allowable $\mathrm{BER}, \mathcal{E}_{t}$, is set to $3 \times 10^{-4}$ and the design target $E_{b} /\left.N_{0}\right|_{t}$ is set to $8 \mathrm{~dB}$ for QPSK signals. For the 16-QAM case, $\mathcal{E}_{t}$ is set to $4 \times 10^{-3}$ and target $E_{b} /\left.N_{0}\right|_{t}$ is set to $10 \mathrm{~dB}$.

Table 2. Simulation parameters.

\begin{tabular}{|c|c|c|}
\hline Scheme & Parameter & Value \\
\hline \multirow{7}{*}{ Common parameters } & Underlying modulation & QPSK/16-QAM \\
\hline & Sub-carriers number, $N$ & 256 \\
\hline & Oversampling factor, $\eta$ & 4 \\
\hline & Reference signal RCM, $\Gamma_{r e f}$ & $1.52 \mathrm{~dB}$ \\
\hline & Cubic metric empirical factor, $\kappa$ & 1.56 \\
\hline & Cubic metric threshold, $\Omega_{0}$ & {$[2.5,6] \mathrm{dB}$} \\
\hline & $E_{b} / N_{0}$ & {$[0,9] \mathrm{dB}$} \\
\hline ICF, SCF & Clipping ratio, $C$ & $6 \mathrm{~dB}$ \\
\hline ICF & Number of iterations, $L$ & 3 \\
\hline \multirow{2}{*}{ SCF } & Equivalency factor, $\lambda$ & 3 \\
\hline & Standard deviation, $\sigma$ & $1 / 2$ \\
\hline SLM & Block size, $U$ & 16 \\
\hline \multirow{3}{*}{ ANN, SNN-CF } & Network Architecture & FFMLP \\
\hline & Output layer activation type & Linear \\
\hline & Normalization & Enabled \\
\hline \multirow{7}{*}{ ANN } & Number of NN modules & 2 \\
\hline & Number of hidden layers, $V$ & 2 \\
\hline & Number of neurons in 1st layer, $h_{1}$ & 2 \\
\hline & $\begin{array}{c}\text { Number of neurons in 2nd layer, } \\
h_{2}\end{array}$ & 1 \\
\hline & Hidden layers activation type & Triangular \\
\hline & Training algorithm & Levenberg-Marquardt \\
\hline & Training epochs & 100 \\
\hline \multirow{7}{*}{ SNN-CF } & Number of NN modules & 1 \\
\hline & Number of hidden layers, $V$ & 2 \\
\hline & Number of neurons in 1st layer, $h_{1}$ & 2 \\
\hline & $\begin{array}{l}\text { Number of neurons in 2nd layer, } \\
h_{2}\end{array}$ & 2 \\
\hline & Hidden layers activation type & Hyperbolic tangent \\
\hline & Training algorithm & Proposed algorithm 2 \\
\hline & Training epochs & 50 \\
\hline
\end{tabular}

The Levenberg-Marquardt algorithm [21] is used to train ANN to emulate the timedomain OFDM signal produced by conventional SCF when fed by the original unmodified OFDM signal at its input. The training epochs defining the number of training iterations 
performed for each input-output sample in the training dataset is configured to the value of 100 iterations for ANN, whereas for the proposed SNN-CF method, we only use 50 updating iterations which is facilitated by virtue of its potentially faster convergence capability.

The dataset batch used for learning the SCF scheme behavioral patterns is complied from a 100 input-output samples. The dataset is split into three parts: (a) the training set, containing $70 \%$ of the dataset samples which are only used during the training stage in which the weights and biases of all layers are found and updated to minimize the MSE over the training set; (b) the testing set, consisting of $15 \%$ of the dataset samples which are used to test the network found by applying the training set and possibly fine-tuning the weights to account for the testing set; and (c) the validation set containing the remaining $15 \%$ of the dataset and is used to validate the trained network's ability to generalize to new data it has never seen before.

\subsection{Results and Discussion}

For QPSK-OFDM signals, the CM reduction performance was investigated for original unmodified OFDM, SLM, SCF, ICF, ANN, and the proposed SNN-CF schemes in terms of the complementary cumulative distribution function, $\mathcal{F}(\Omega)=\operatorname{Pr}\left\{\Omega>\Omega_{0}\right\}$, for different cubic metric threshold $\left(\Omega_{0}\right)$ levels ranging from 2.5 to $6 \mathrm{~dB}$ as shown in Figure 6a. The proposed SNN-CF scheme outperforms the best of the three conventional approaches and shows more than a $0.5 \mathrm{~dB}$ performance improvement compared to ANN at CCDF levels as low as $10^{-3}$. Similarly, Figure $6 \mathrm{~b}$ shows that the proposed SNN-CF scheme maintains its superior CM reduction performance for the higher order 16-QAM-OFDM case. This is accomplished by scanning the neighborhood of SCF during the training stage to find an SNN-CF network with the highest possible performance.

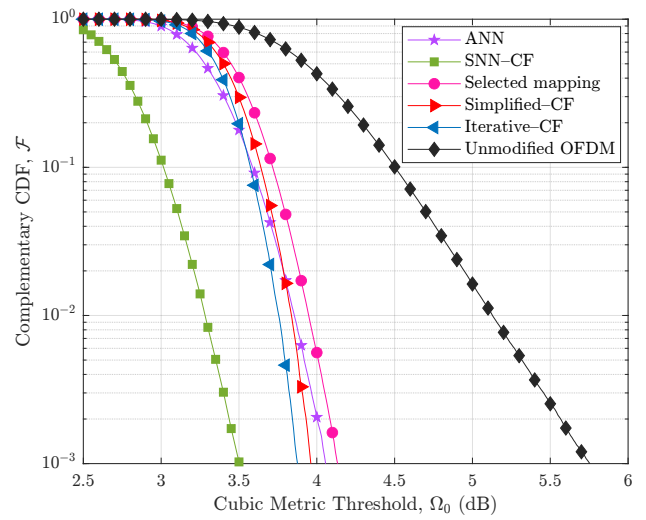

(a)

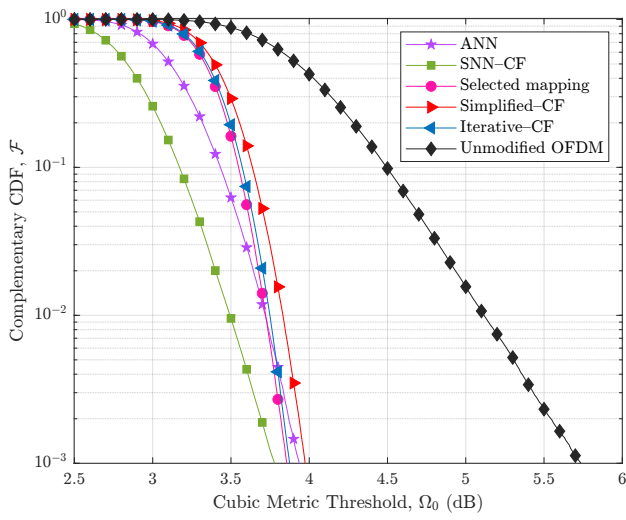

(b)

Figure 6. CM reduction performance of the proposed SNN-CF vs. ANN [12], selected mapping [4], simplified-CF [8], iterative-CF [5], and unmodified OFDM for (a) QPSK-OFDM, and (b) 16-QAM-OFDM.

The proposed SNN-CF exhibits lower OoB power leakage than ANN for both QPSK and 16-QAM modulations, as shown in Figure 7. This figure plots the power spectral density (PSD) curves of OFDM signals as a function of normalized frequency. Only original OFDM is included as a baseline for comparison since SLM has identical power distribution as the original OFDM and conventional ICF and SCF are both assumed ideal, rendering their PSD performance matching with the original unprocessed OFDM. In the neighborhood of the in-band, both ANN and the proposed SNN-CF show similar leakage for QPSK-OFDM as shown in Figure 7a. However, the OoB spectrum radiation caused by the proposed SNN-CF scheme is rapidly diminished compared to ANN, facilitating more relaxed guard-band requirements between different operators. For 16-QAM-OFDM, OoB power leakage of the proposed SNN-CF scheme is consistently lower than ANN, as shown in Figure $7 \mathrm{~b}$. This is mainly because the proposed SNN-CF is able to learn the amplitude variations of SCF more accurately than ANN. 


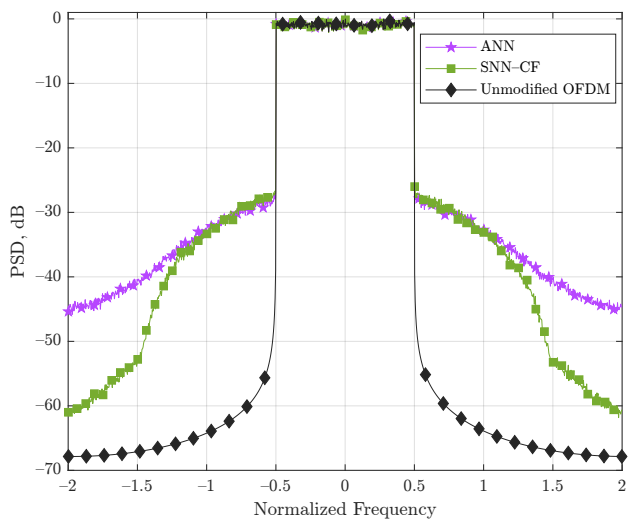

(a)

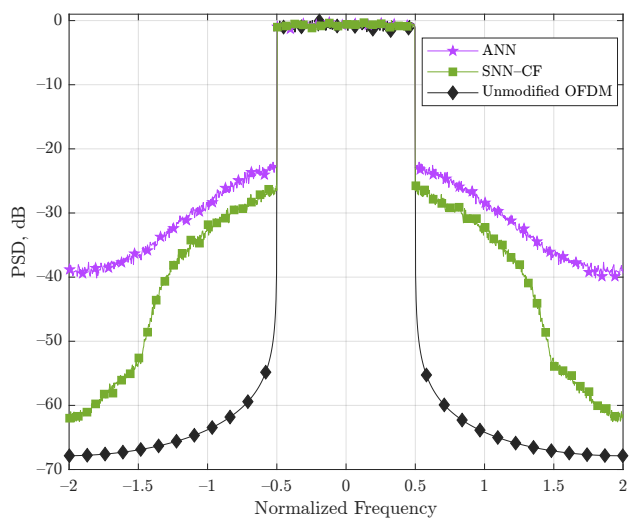

(b)

Figure 7. Power leakage performance of the proposed SNN-CF vs. ANN [12] and unmodified OFDM in terms of normalized PSD vs. normalized frequency for (a) QPSK-OFDM; and (b) 16-QAM-OFDM modulation.

The BER performance of all QPSK-based schemes are compared in Figure 8a for $E_{b} / N_{0}$ ranging from 0 to $9 \mathrm{~dB}$. As expected, SLM preserves the signal integrity and results in the lowest possible BER which is identical to the BER performance of original OFDM. SCF and ICF come next and are closely followed by ANN and the proposed SNN-CF scheme. For 16-QAM-OFDM transmissions, the proposed SNN-CF scheme remains remarkably close to conventional SCF in terms of BER performance, as shown in Figure 8b. Although ANN maintains its CM reduction performance, it starts to exhibit a deteriorated BER performance resulting in about a $3 \mathrm{~dB}$ performance gap from conventional SCF at the $10^{-3}$ BER level. In contrast, the proposed SNN-CF scheme remains within approximately $0.3 \mathrm{~dB}$ from conventional SCF at the same BER level. This is facilitated by the more accurate representation of SNN-CF to SCF signals.

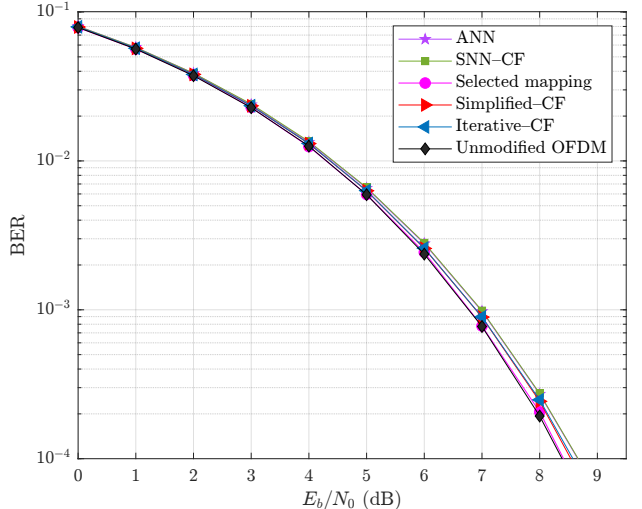

(a)

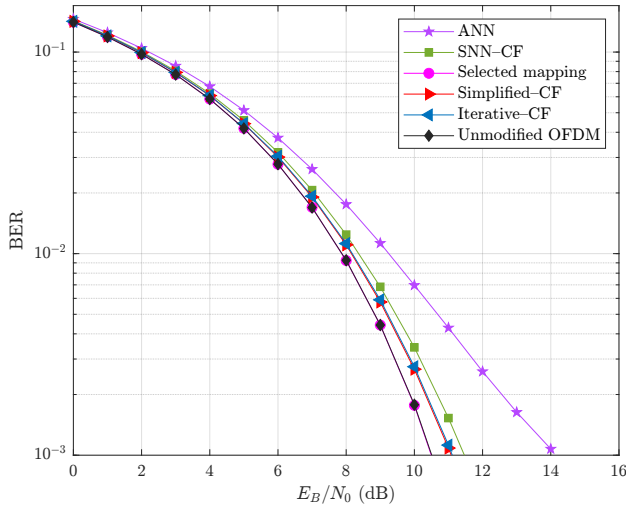

(b)

Figure 8. BER performance of the proposed SNN-CF vs. ANN [12], selected mapping [4], simplifiedCF [8], iterative-CF [5], and unmodified OFDM for (a) QPSK-OFDM; and (b) 16-QAM-OFDM.

Figure 9a plots the BER performance of the proposed SNN-CF scheme vs. ANN, SLM, SCF, ICF, and raw OFDM signals for 16-QAM-based OFDM transmissions deploying a non-linear solid state HPA with the signal envelope amplitude, $E$, undergoing an amplitudeto-amplitude (AM/AM) conversion accomplished by the transfer function [22]:

$$
f(E)=\frac{g E}{\left(1+\left(g \frac{E}{E_{0}}\right)^{2 R}\right)^{1 / 2 R}}
$$


where $g$ is the small-signal gain, $E_{0}$ is the output limiting parameter, and $R$ is a parameter that controls the transition rate from the linear operation region to the limiting operation region. These parameters are set to $g=1, E_{0}=1$, and $P=3$ as in [22]. Although ANN lags behind SCF and ICF with a performance gap exceeding $3 \mathrm{~dB}$ at the $10^{-3}$ mark, the proposed scheme manages to maintain a close proximity to within $0.4 \mathrm{~dB}$ of SCF and ICF at the same level.

Figure $9 \mathrm{~b}$ retraces the PSD performance of ANN and the proposed SNN-CF scheme along with the benchmark schemes and unmodified OFDM signals when the solid state HPA is deployed. SLM, SCF, and ICF provide more controlled OoB radiations in the neighborhood of the in-band, where the input back-off of HPA and clipping ratio are set to 3.5 and $6 \mathrm{~dB}$, respectively. Both ANN and SNN-CF exhibit a steady roll-off decline in the neighborhood of the in-band with an almost consistent leakage gap of about $3 \mathrm{~dB}$ in favor of SNN-CF. In addition, as we move further away, the gap between ANN and the proposed SNN-CF scheme grows rapidly as ANN roll-off slows down whereas the OoB leakage of the proposed scheme decays significantly and goes below the benchmarks. Although SCF provides better overall PSD performance than the proposed SNN-CF, it requires a noticeably higher processing complexity.

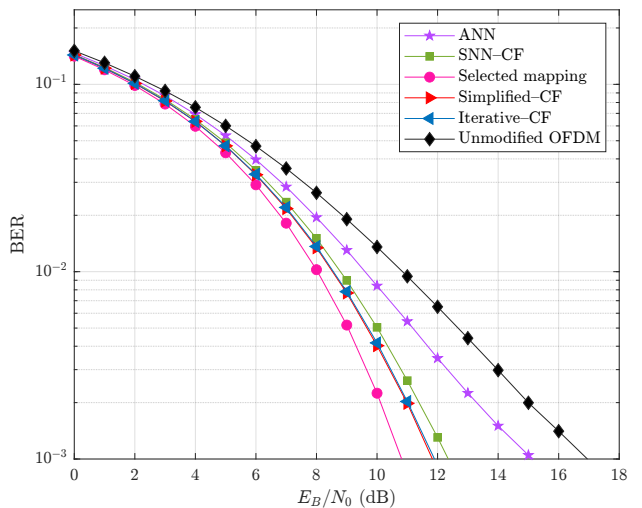

(a)

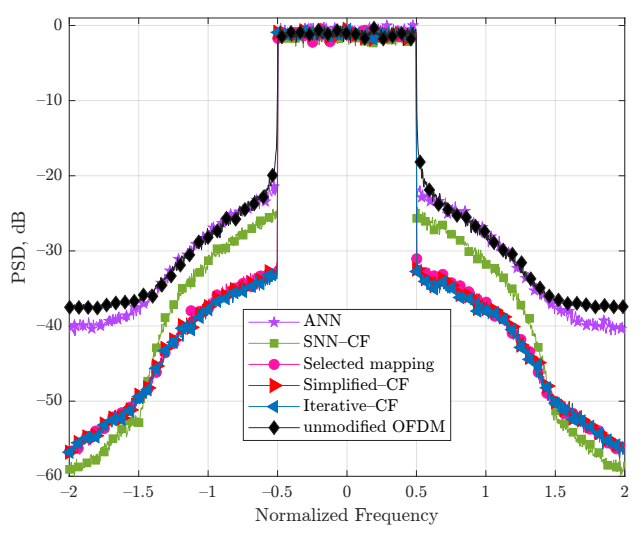

(b)

Figure 9. Performance evaluation for 16-QAM-OFDM transmissions deploying non-linear HPA with $3.5 \mathrm{~dB}$ input back-off and $6 \mathrm{~dB}$ clipping ratio: (a) BER; and (b) PSD.

Figure 10 shows an alternative view on OoB power leakage of ANN and the proposed SNN-CF for QPSK and 16-QAM. The normalized out-of-band leakage power (NOLP) $\gamma$ is defined as

$$
\gamma \triangleq 10 \log _{10}\left(P_{l} / P_{\text {in }}\right) d B,
$$

where $P_{l}$ is the total leaked power and $P_{i n}$ is the power residing within the in-band. Lower NOLP levels are desirable for superior power leakage performance. Therefore, we use the complementary CDF on different threshold values of the NOLP: $\mathcal{F}(\gamma) \triangleq \operatorname{Pr}\left\{\gamma>\gamma_{0}\right\}$. The threshold level $\gamma_{0}$ is varied within the ranges $[-42,-24] \mathrm{dB}$ and $[-42,-22] \mathrm{dB}$ for QPSK and 16-QAM, respectively, in order to capture the OoB leakage performance from CCDF unity level (corresponding to uncrossable threshold points) to levels as low as $10^{-3}$ (corresponding to crossable threshold points with $99.9 \%$ success rate). As shown in Figure 10a, the proposed SNN-CF scheme generally outperforms ANN in terms of the reduction achieved on NOLP for QPSK modulation with a performance gap of about $4.25 \mathrm{~dB}$ at CCDF level of $10^{-3}$. In addition, for the 16-QAM case, the proposed SNNCF scheme exhibits a much more controlled power leakage than ANN does with an approximately $7.25 \mathrm{~dB}$ performance gap at the $10^{-3}$ CCDF mark, as shown in Figure 10b. This superior performance of SNN-CF compared to ANN is the result of the more accurate peak-tracking of SCF signals, as modeled by the non-linear SNN-CF mapper. 


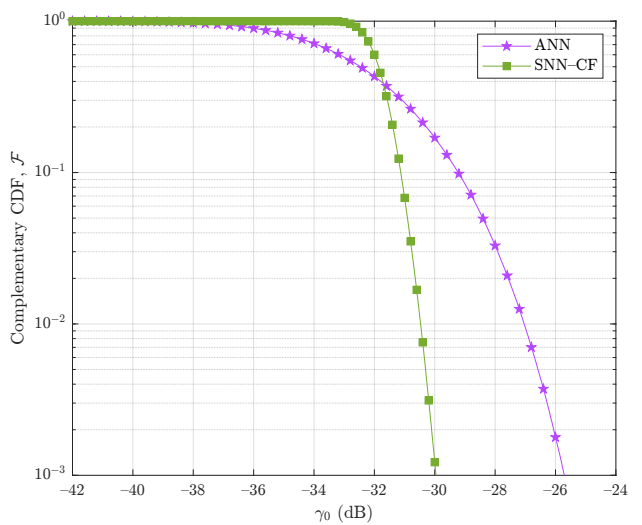

(a)

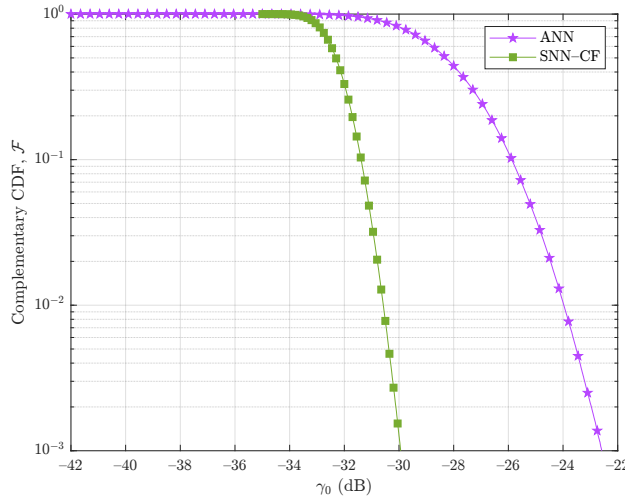

(b)

Figure 10. Power leakage performance of the proposed SNN-CF vs. ANN [12] in terms of the reduction achieved in terms of the outband-to-inband power ratio for (a) QPSK-OFDM; and (b) 16QAM-OFDM.

Figure 11 investigates the BER performance of the proposed SNN-CF scheme for higher order QAM modulations. For all three cases of 64-QAM, 256-QAM, and 1024QAM transmissions, the proposed SNN-CF scheme successfully remains within close performance to original OFDM. For example, the performance gap for 64-QAM-based operation is about $1.25 \mathrm{~dB}$ at a BER level of $10^{-3}$. Similarly, for the 256-QAM and 1024-QAM cases, the performance gaps at the same BER level are about 1.5 and $1.75 \mathrm{~dB}$, respectively. To attain even narrower gaps for higher modulation orders, the underlying neural network module can be expanded by slightly increasing the neurons within the layers, for example, to maintain the complexity within reasonable bounds.

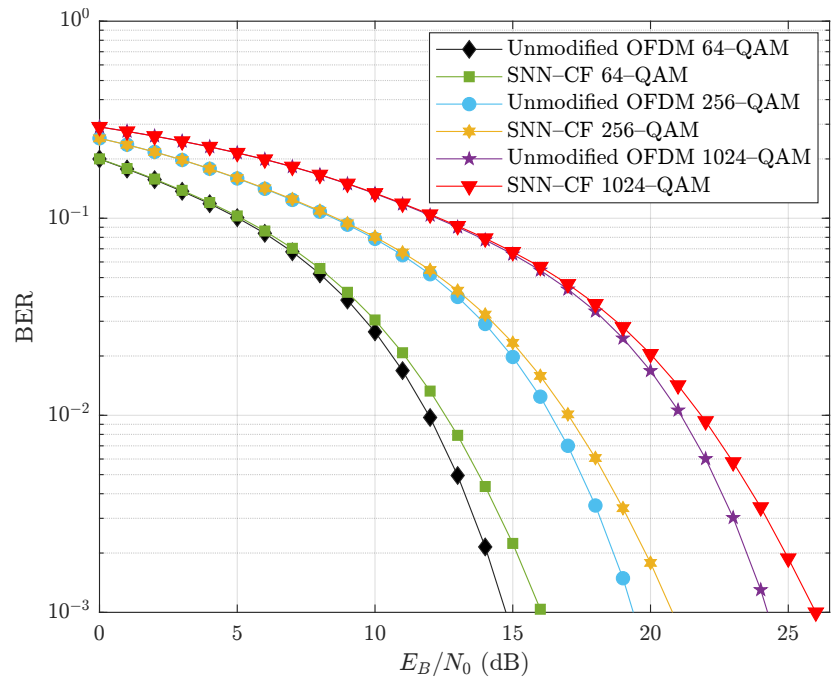

Figure 11. BER performance tracking of the proposed SNN-CF scheme for higher order modulations: 64-QAM, 256-QAM, and 1024-QAM.

\section{Conclusions}

In this paper, we proposed a novel neural network-based approach for PAPR reduction in OFDM signals with common conventional techniques for benchmark comparison. To surpass the limits of conventional solutions, we proposed a novel network-selection algorithm that scans non-linear maps in the neighborhood of conventional CF-based schemes to find the network with the lowest cubic metric reduction for certain QoS requirements. Comparative analysis in terms of the complexity required for processing OFDM signals showed significant improvement in favor of NN approaches which makes them more suitable for applications with tight low-latency real-time operation requirements. Even 
though our proposed SNN-CF scheme incurs a slightly higher computational cost than $\mathrm{ANN}$, it provided the highest performance in terms of $\mathrm{CM}$ reduction while maintaining the $\mathcal{O}(N)$ complexity class of ANN. In addition, unlike ANN, the proposed scheme maintains much closer BER performance to conventional CF schemes at higher modulations. Finally, the proposed scheme exhibits less OoB radiations than ANN making it more desirable for interference-sensitive transmission environments such as heterogeneous networks.

Author Contributions: Conceptualization, A.G. and O.M.; methodology, A.G.; software, A.G.; validation, A.G. and O.M.; formal analysis, A.G. and O.M.; investigation, A.G. and O.M.; resources, O.M.; writing—original draft, A.G.; writing—review and editing, O.M.; visualization, O.M.; supervision, O.M.; funding acquisition, O.M. All authors have read and agreed to the published version of the manuscript.

Funding: This work was supported in part by the Support Center for Advanced Telecommunications Technology Research Foundation. In addition, Ahmad Gendia is funded by a full scholarship from the Ministry of Higher Education of the Arab Republic of Egypt.

Conflicts of Interest: The authors declare no conflict of interest associated with this manuscript.

\section{References}

1. Muller, S.H.; Huber, J.B. OFDM with reduced peak-to-average power ratio by optimum combination of partial transmit sequences. Electron. Lett. 1997, 33, 368-369. [CrossRef]

2. Tellado, J.; Cioffi, J.M. Peak Power Reduction for Multicarrier Transmission. Available online: http:/ / citeseerx.ist.psu.edu/ viewdoc/summary?doi=10.1.1.55.3075 (accessed on 15 July 2021)

3. Anoh, K.; Adebisi, B.; Rabie, K.M.; Tanriover, C. Root-Based Nonlinear Companding Technique for Reducing PAPR of Precoded OFDM Signals. IEEE Access 2018, 6, 4618-4629. [CrossRef]

4. Breiling, H.; Muller-Weinfurtner, S.H.; Huber, J.B. SLM peak-power reduction without explicit side information. IEEE Commun. Lett. 2001, 5, 239-241. [CrossRef]

5. Armstrong, J. Peak-to-average power reduction for OFDM by repeated clipping and frequency domain filtering. Electron. Lett. 2002, 38, 246-247. [CrossRef]

6. Wang, Y.; Luo, Z. Optimized Iterative Clipping and Filtering for PAPR Reduction of OFDM Signals. IEEE Trans. Commun. 2011, 59, 33-37. [CrossRef]

7. Deng, S.; Lin, M. Recursive Clipping and Filtering with Bounded Distortion for PAPR Reduction. IEEE Trans. Commun. 2007, 55, 227-230. [CrossRef]

8. Wang, L.; Tellambura, C. A simplified clipping and filtering technique for PAPR reduction in OFDM systems. IEEE Signal Process. Lett. 2005, 12, 453-456. [CrossRef]

9. Baxley, R.J.; Zhao, C.; Zhou, G.T. Constrained Clipping for Crest Factor Reduction in OFDM. IEEE Trans. Broadcast. 2006, 52, 570-575. [CrossRef]

10. Anoh, K.; Tanriover, C.; Adebisi, B. On the Optimization of Iterative Clipping and Filtering for PAPR Reduction in OFDM Systems. IEEE Access 2017, 5, 12004-12013. [CrossRef]

11. Liu, X.; Zhang, X.; Xiong, J.; Gu, F.; Wei, J. An Enhanced Iterative Clipping and Filtering Method Using Time-Domain Kernel Matrix for PAPR Reduction in OFDM Systems. IEEE Access 2019, 7, 59466-59476. [CrossRef]

12. Sohn, I.; Kim, S.C. Neural Network Based Simplified Clipping and Filtering Technique for PAPR Reduction of OFDM Signals. IEEE Commun. Lett. 2015, 19, 1438-1441. [CrossRef]

13. Yamashita, K.; Ohta, M.; Jiang, W. Reducing peak-to-average power ratio of multicarrier modulation by Hopfield neural network. Electron. Lett. 2002, 38, 1370-1371. [CrossRef]

14. Hopfield, J.J.; Tank, D.W. Neural computation of decisions in optimization problems. Biol. Cybern. 1985, 52, 141-152. [CrossRef] [PubMed]

15. Sohn, I. RBF Neural Network Based SLM Peak-to-Average Power Ratio Reduction in OFDM Systems. ETRI J. 2007, 29, 402-404. [CrossRef]

16. Jabrane, Y.; Jiménez, V.P.G.; Armada, A.G.; Said, B.A.E.; Ouahman, A.A. Reduction of Power Envelope Fluctuations in OFDM Signals by using Neural Networks. IEEE Commun. Lett. 2010, 14, 599-601. [CrossRef]

17. Sohn, I. A Low Complexity PAPR Reduction Scheme for OFDM Systems via Neural Networks. IEEE Commun. Lett. 2014, 18, 225-228. [CrossRef]

18. Kim, M.; Lee, W.; Cho, D. A Novel PAPR Reduction Scheme for OFDM System Based on Deep Learning. IEEE Commun. Lett. 2018, 22, 510-513. [CrossRef]

19. Technical Report R1-060023: Cubic Metric in 3GPP-LTE. 3GPP Technical Specification Group Radio Access Network WG1. Available online: https://www.3gpp.org/ftp/tsg_ran/wg1_rl1/TSGR1_AH/LTE_AH_0601/Docs/ (accessed on 15 July 2021) 
20. Kageyama, T.; Muta, O.; Gacanin, H. Performance Analysis of OFDM with Peak Cancellation Under EVM and ACLR Restrictions. IEEE Trans. Veh. Technol. 2020, 69, 6230-6241. [CrossRef]

21. Marquardt, D.W. An Algorithm for Least-Squares Estimation of Nonlinear Parameters. J. Soc. Ind. Appl. Math. 1963, 11, 431-441. [CrossRef]

22. Rapp, C. Effects of HPA-nonlinearity on a 4-DPSK/OFDM-signal for a digital sound broadcasting signal. ESA Spec. Publ. 1991, 332, 179-184. 